\title{
A Linac simulation code for macro-particles tracking and steering algorithm implementation
}

\author{
Yipeng Sun \\ SLAC National Accelerator Laboratory, Menlo Park, California 94025, USA
}

May 2, 2012

\begin{abstract}
In this paper, a linac simulation code written in Fortran90 is presented and several simulation examples are given. This code is optimized to implement linac alignment and steering algorithms, and evaluate the accelerator errors such as RF phase and acceleration gradient, quadrupole and BPM misalignment. It can track a single particle or a bunch of particles through normal linear accelerator elements such as quadrupole, RF cavity, dipole corrector and drift space. One-to-one steering algorithm and a global alignment (steering) algorithm are implemented in this code.
\end{abstract}

\section{Overview}

In a linear accelerator, one outstanding problem is to align the accelerator components or steer the beam, in order to preserve the beam condition (such as transverse emittance). After the initial survey of the linac components which usually achieves mm range accuracy, there are several beambased alignment (BBA) techniques that could be applied to improve the alignment.

A linac simulation code is written in Fortran90 which achieves some general functions such as particles tracking, beam and lattice error generation and addition. At the mean time this code is optimized for linac alignment or steering algorithm implementation. Some study results using this simulation code can be found in [1] [2] [3].

The basic modules of this simulation code are listed here which will be discussed in details in the following sections.

- General setup

- Linac model

- Error generation and addition

- Single particle tracking

- Macro-particle tracking

- Post processing 


\section{General setup}

In this module, initial beam parameters are read in, such as beam energy, transverse emittance, RMS energy spread and bunch length. One could generate a Gaussian or Uniform distribution of the bunch. Other parameters such as RMS quadrupole misalignment, RMS BPM to quadrupole offset, RMS BPM resolution, initial beam offset and angle are also specified and generated in this module.

One can choose to use either single particle mode or bunch mode here. Single bunch mode and multi bunch mode are also available.

The linac lattice (sequence) is read in by this module which is an element-by-element definition with the key parameters of each component.

\section{$3 \quad$ Linac model}

There are two available methods to model a linac lattice, which are thin lens kick method and matrix mode. All momentum-dependent higher order terms are kept in thin lens kick mode. In the matrix mode, basic linac components such as quadrupole, RF cavity, dipole corrector and drift are modeled as first order thin lens.

The transfer of the particle's coordinates are (up to first-order) shown below, where $R$ denotes the first order transfer matrix between initial and final locations.

$$
\left(\begin{array}{c}
x \\
x^{\prime} \\
y \\
y^{\prime} \\
z \\
\delta
\end{array}\right)=\mathbf{R} \cdot\left(\begin{array}{c}
x_{0} \\
x_{0}^{\prime} \\
y_{0} \\
y_{0}^{\prime} \\
z_{0} \\
\delta_{0}
\end{array}\right)
$$

\subsection{Quadrupole}

The 6 by 6 transport matrix of a quadrupole magnet with integrated strength $K_{1}$ can be written as

$$
\mathbf{R}_{\text {Quad }}=\left(\begin{array}{cccccc}
1 & 0 & 0 & 0 & 0 & 0 \\
-K_{1} & 1 & 0 & 0 & 0 & 0 \\
0 & 0 & 1 & 0 & 0 & 0 \\
0 & 0 & K_{1} & 1 & 0 & 0 \\
0 & 0 & 0 & 0 & 1 & 0 \\
0 & 0 & 0 & 0 & 0 & 1
\end{array}\right)
$$

\section{$3.2 \quad$ RF cavity}

The 6 by 6 transport matrix of an RF cavity with length $L_{R F}$ and energy ratio $a=\frac{E_{0}}{E_{f}}$ can be written as 


$$
\mathbf{R}_{\mathbf{R F}}=\left(\begin{array}{cccccc}
1 & L_{R F} & 0 & 0 & 0 & 0 \\
0 & a & 0 & 0 & 0 & 0 \\
0 & 0 & 1 & L_{R F} & 0 & 0 \\
0 & 0 & 0 & a & 0 & 0 \\
0 & 0 & 0 & 0 & 1 & 0 \\
0 & 0 & 0 & 0 & 0 & a
\end{array}\right)
$$

\subsection{Dipole corrector}

The 6 by 6 transport matrix of the normal dipole magnet can be written as

$$
\mathbf{R}_{\mathbf{B}(\theta, \rho)}=\left(\begin{array}{cccccc}
\cos \theta & \rho \sin \theta & 0 & 0 & 0 & \rho(1-\cos \theta) \\
-\sin \theta / \rho & \cos \theta & 0 & 0 & 0 & \sin \theta \\
0 & 0 & 1 & \rho \theta & 0 & 0 \\
0 & 0 & 0 & 1 & 0 & 0 \\
-\sin \theta & \rho(\cos \theta-1) & 0 & 0 & 1 & \rho(\sin \theta-\theta) \\
0 & 0 & 0 & 0 & 0 & 1
\end{array}\right)
$$

\subsection{Drift}

The 6 by 6 transport matrix of a drift with length $L$ can be written as

$$
\mathbf{R}_{\text {Drift }}=\left(\begin{array}{cccccc}
1 & L & 0 & 0 & 0 & 0 \\
0 & 1 & 0 & 0 & 0 & 0 \\
0 & 0 & 1 & L & 0 & 0 \\
0 & 0 & 0 & 1 & 0 & 0 \\
0 & 0 & 0 & 0 & 1 & 0 \\
0 & 0 & 0 & 0 & 0 & 1
\end{array}\right)
$$

\section{Error generation and addition}

Random and systematic errors can be generated and then added on all the linac elements, such as RF phase and amplitude, linac components offset and tilt, higher order magnetic fields of quadrupoles, sextupoles and dipoles. Here an example for higher order fields error is given. A general formula for higher order fields (with normal and skew components) is listed below with the normalized magnet multipole strength defined.

$$
B_{y}+i B_{x}=B \sum_{n=1}^{10}\left(b_{n}-i a_{n}\right)\left(\frac{x}{r_{0}}+i \frac{y}{r_{0}}\right)^{n-1}
$$

where $B$ denotes the magnetic field on the reference trajectory, $b_{n}$ the normal multipole coefficient, and $a_{n}$ the skew multipole coefficient. The index $n$ is associated with the $2(n+1)$ pole.

$$
K_{n}=\frac{\partial^{n} B / \partial x^{n} \cdot L}{B \rho}
$$


where $K_{n}$ denotes the normalized magnet multipole strength, $\partial^{n} B / \partial x^{n}$ field gradient, $L$ magnetic length of this element, and $B \rho$ the beam rigidity.

Other types of error such as magnetic field scale error and BPM scale error can also be included. Feedback systems can also be included in the simulation such as to maintain constant energy gain over the linac.

\section{$5 \quad$ Single particle or Macro-particle tracking}

In the thin lens kick mode, an element-by-element tracking procudure is adopted for both single particle and macro-particle tracking. Meanwhile for matrix mode, one could either track elementby-element or calculate the overall transfer matrix directly.

For single particle tracking mode, one could specify 6-D coordinates up to 20 particles. For Macro-particle tracking mode, one could choose single bunch or multi bunch mode. Gaussian or Uniform distribution are available internally, and external input files can also be read in as initial coordinates. Beam parameters can be varied between different bunches in the multi bunch mode.

\section{Post processing}

In post processing module, statistical calculation is performed on beam parameters and lattice parameters. Beam trajectory and RMS beam size can be calculated. 'TWISS' parameters at different linac locations could also be calculated using the bunch coordinates, such as beta functions and dispersion functions. Projected emittance and linear dispersion subtracted emittance can then be calculated using these beam-induced 'TWISS' parameters or by using design optics 'TWISS' parameters.

\section{Simulation results examples}

In this section several simulation examples are presented (from Figure 1 to Figure 10), to illustrate the functions of this simulation code.

\section{Acknowledgement}

The author would like to thank C. Adolphsen for helpful discussions.

This work was supported by the DOE under Contract DE-AC02-76SF00515.

\section{References}

[1] Yipeng Sun, 'Linac Alignment Algorithm:1-to-1 Steering,' SLAC-PUB-14322 (2011).

[2] Yipeng Sun, 'Emittance Growth in the NLCTA First Chicane,' SLAC-PUB-14323 (2011).

[3] Yipeng Sun, 'A New Linac Steering Algorithm,' SLAC-PUB-14466 (2012). 

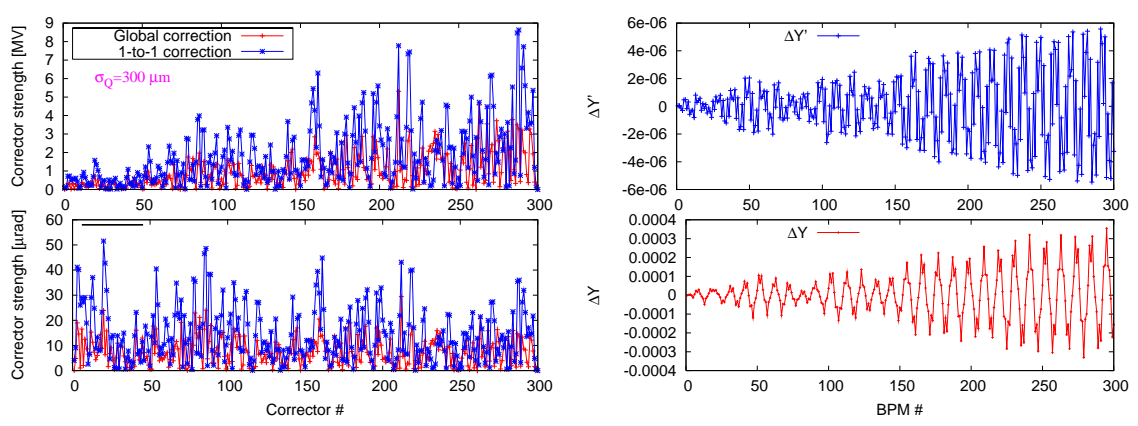

Figure 1: Left: dipole corrector strength; Right: difference trajectory.
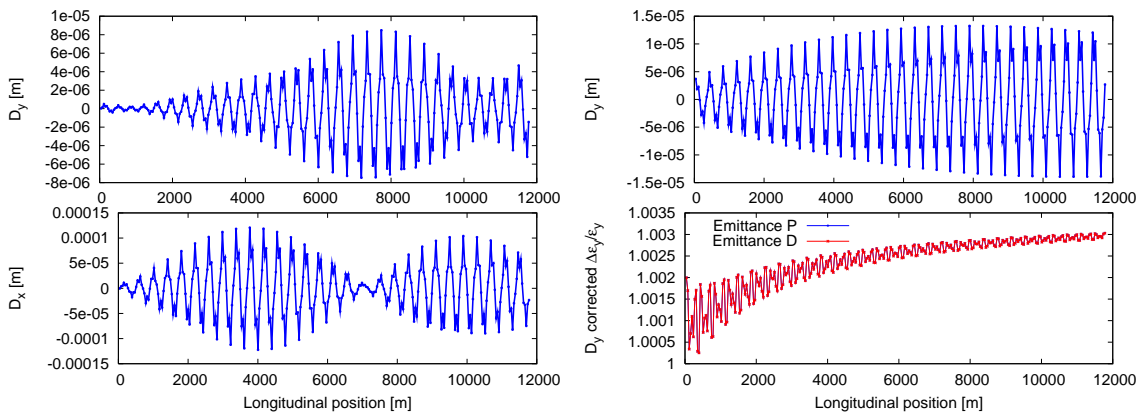

Figure 2: Left: dispersion; Right: dispersion and emittance.
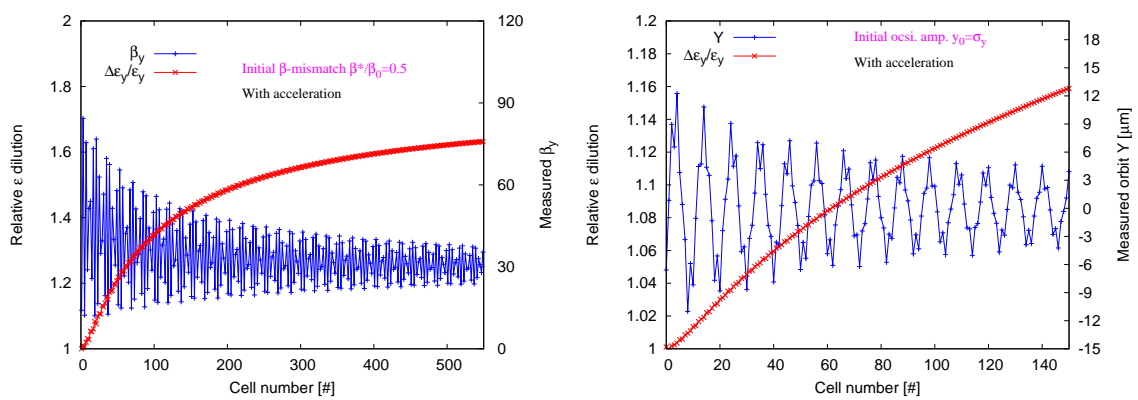

Figure 3: Left: Filamentation from initial beta mismatch; Right: Filamentation from initial trajectory offset.
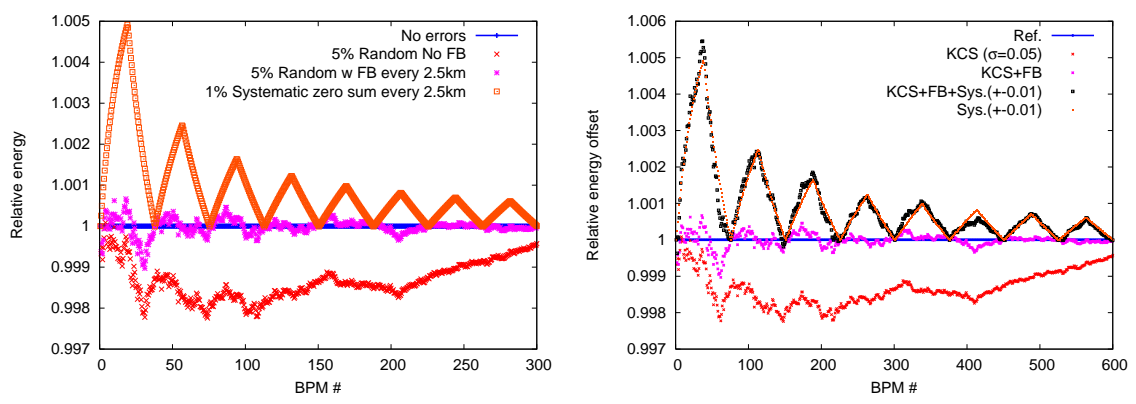

Figure 4: Left: energy error along linac; Right: energy error along linac with klystron cluster. 

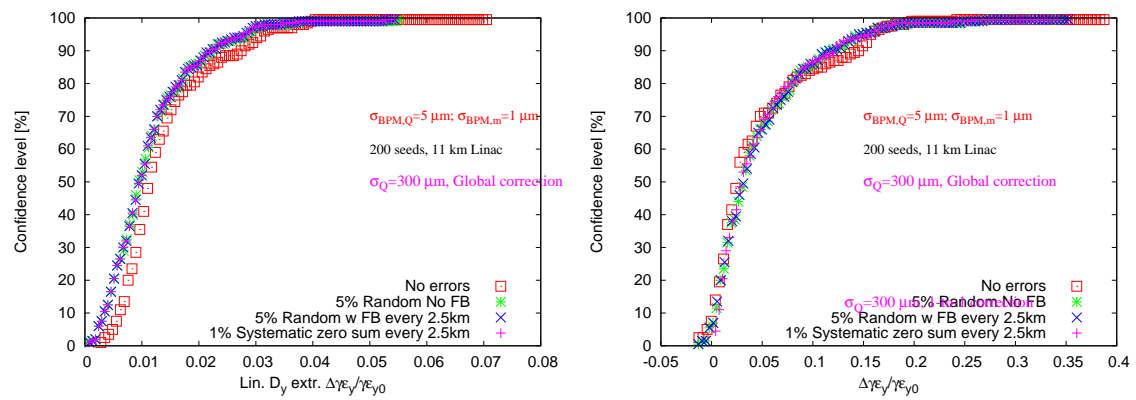

Figure 5: Left: confidence level for linear dispersion subtracted emittance; Right: confidence level for projected emittance.
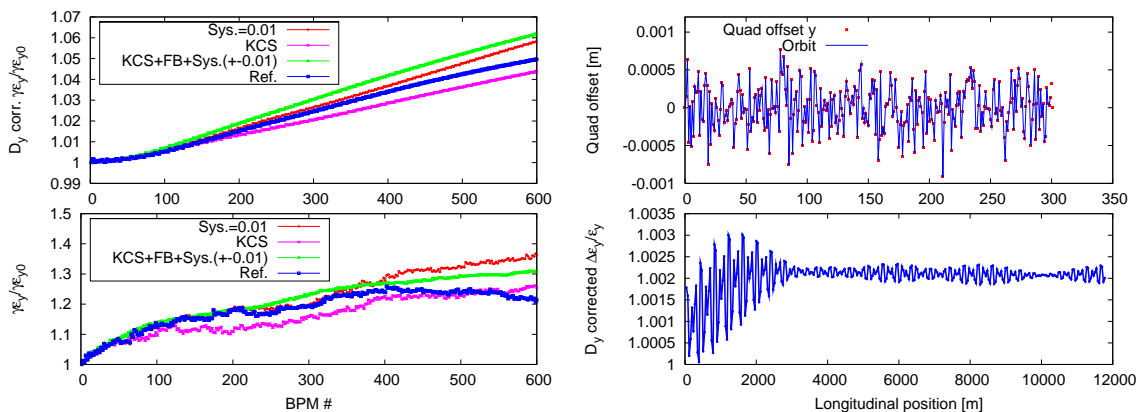

Figure 6: Left: emittance growth with KCS and 1-to-1 steering etc.; Right: emittance growth and trajectory.
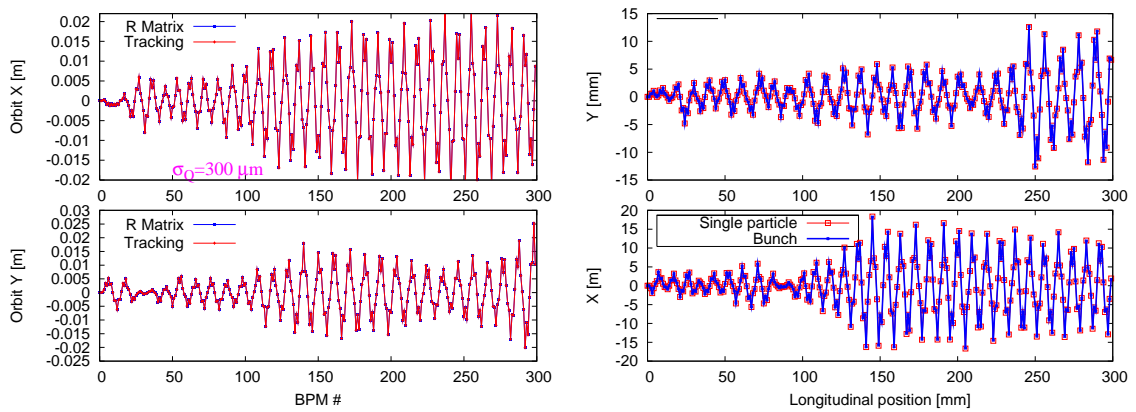

Figure 7: Left: benchmark trajectory $\mathrm{R}$ matrix and tracking; Right: benchmark trajectory single particle and bunch.
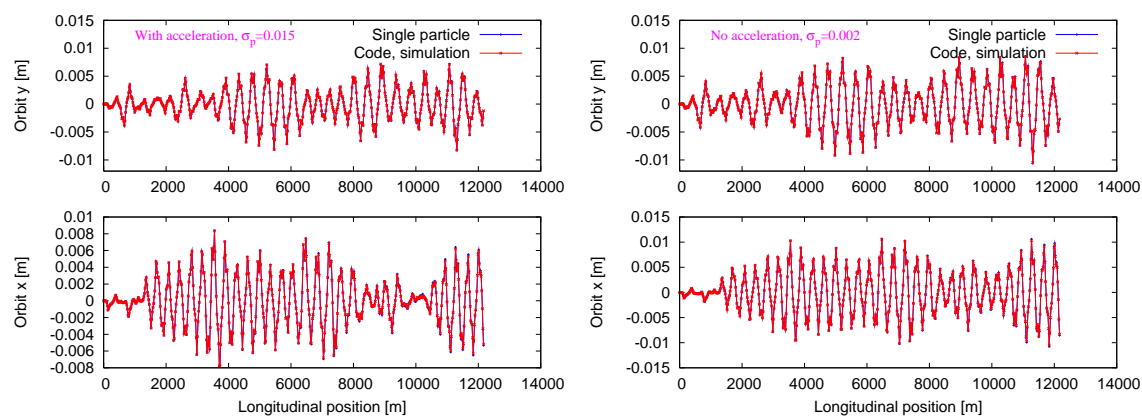

Figure 8: Left: benchmark trajectory with acceleration; Right: benchmark trajectory without acceleration. 

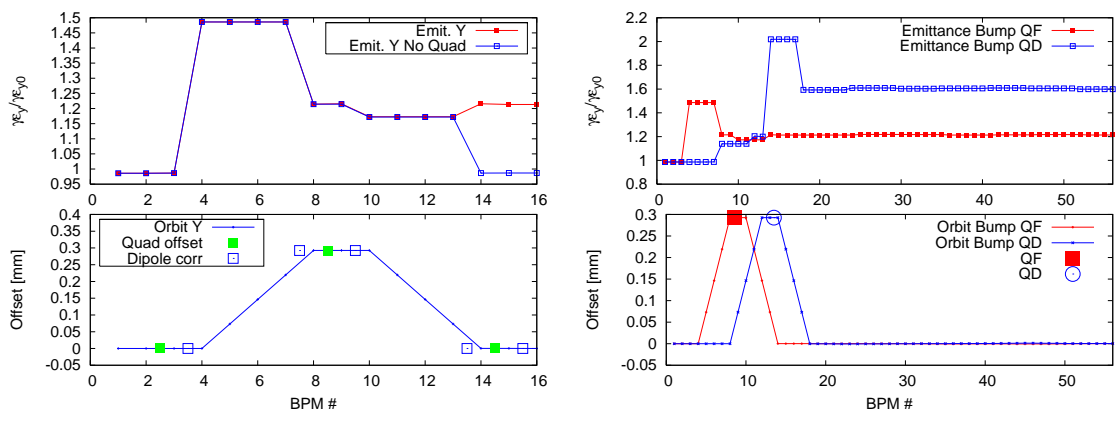

Figure 9: Left: illustration emittance growth in single cell; Right: illustration emittance growth with emittance bump.
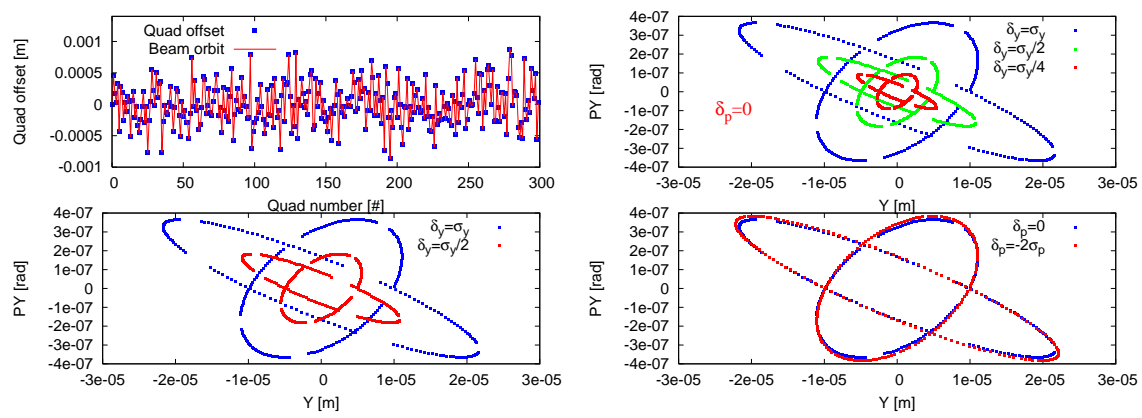

Figure 10: Left: phase space and trajectory; Right: phase space.
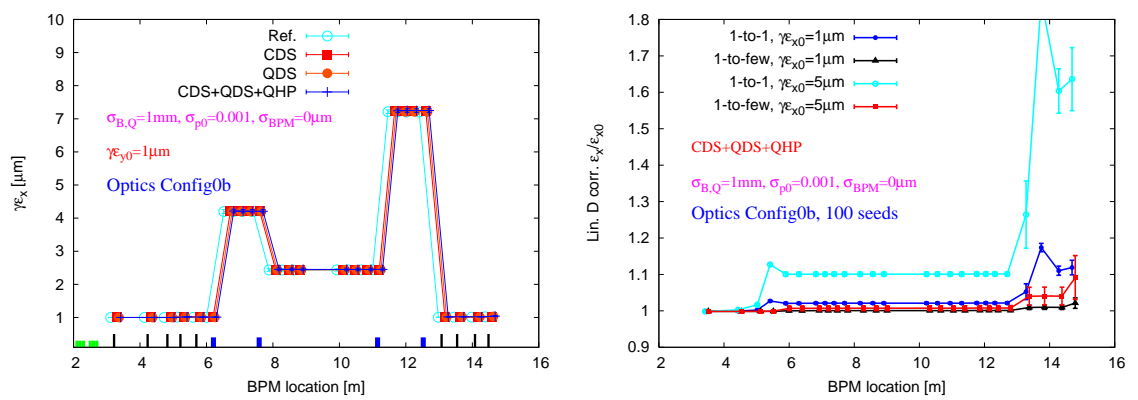

Figure 11: NLCTA emittance growth with higher order magnetic errors. Left: with one-to-few steering; Right: with one-to-one steering. 


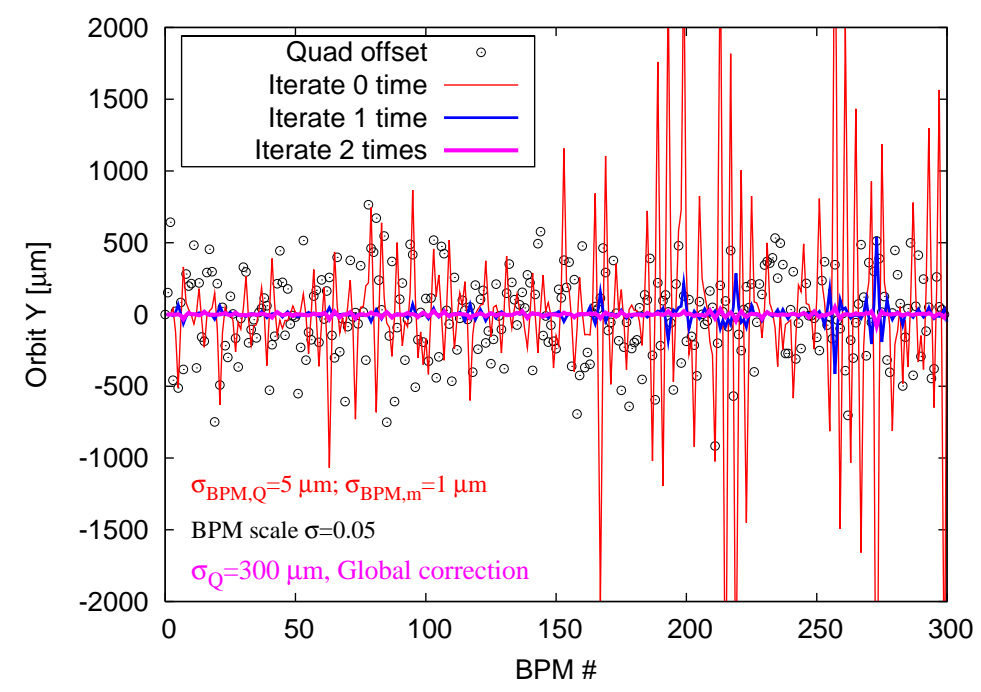

Figure 12: With global steering algorithm applied, iterate to minimize beam trajectory. 